\title{
Impact of riboflavin status on haemoglobin and risk of anaemia in pregnancy
}

\author{
B. Duffy ${ }^{1}$, K. Pentieva ${ }^{1}$, M. Ward ${ }^{1}$, E. Psara ${ }^{1}$, E. O’Sullivan ${ }^{1}$, G. Horrigan ${ }^{1}$, K. Glackin ${ }^{2}$, \\ L. Doherty and H. McNulty ${ }^{1}$ \\ ${ }^{1}$ Nutrition Innovation Centre for Food and Health (NICHE), School of Biomedical Sciences, Ulster University, \\ Coleraine, BT52 1SA, UK, \\ ${ }^{2}$ Western Health \& Social Care Trust, UK and \\ ${ }^{3}$ Northern Health \& Social Care Trust, UK
}

Anaemia affects $42 \%$ of pregnancies worldwide and is associated with a number of adverse maternal and neonatal pregnancy outcomes including postpartum haemorrhage, preterm delivery, stillbirth and reduced offspring birthweight. ${ }^{(1,2)}$ Whilst iron deficiency is the most common nutritional cause of anaemia in pregnancy, low status of the B vitamin, riboflavin, may also be implicated due to its role in iron metabolism, (specifically in the flavin-dependent release of stored iron for erythropoiesis), but this is rarely investigated in human studies. ${ }^{(3)}$ Low or deficient riboflavin status occurs more commonly among women of reproductive age than is generally recognised, and studies to date investigating the association between riboflavin status and haemoglobin (Hb) in pregnancy have been largely confined to low-middle income populations. ${ }^{(4)}$ The aim of this study was to examine the associations of riboflavin with $\mathrm{Hb}$, and to determine the role of riboflavin as a predictor of anaemia during pregnancy.

Data for this analysis were obtained from healthy pregnant women in Northern Ireland and the Republic of Ireland, enrolled on the ongoing Optimal Nutrition for the Prevention of Hypertension in Pregnancy using a Personalised Approach (OptiPREG) study. Detailed health, dietary and lifestyle information, along with a blood sample for analysis of B vitamin biomarkers and haematological measures, were obtained from all participants at the 12th gestational week $(\mathrm{GW})(n=2,153)$ and at the 36 th $\mathrm{GW}$ (in a subset of mothers; $n=372$ ). Riboflavin status was determined by the functional assay, erythrocyte glutathione reductase activation coefficient (EGRac), whereby higher values indicate lower riboflavin status.

Biomarker analysis showed that $68 \%$ of pregnant women had low or deficient riboflavin status. Linear regression analysis found riboflavin status to be a significant determinant of $\mathrm{Hb}$ at the 12 th $\mathrm{GW}(\beta=-0.128, \mathrm{P}=0.001)$, whilst the odds of developing anaemia at the 12th GW increased with decreasing riboflavin status $(\beta=2.4, \mathrm{OR}: 10.9, \mathrm{CI}: 2.2-53.3, \mathrm{P}=0.003)$. Hb was $0.32 \mathrm{~g} / \mathrm{dl}$ lower at 12 th $\mathrm{GW}(\mathrm{P}=0.026)$, and $0.64 \mathrm{~g} / \mathrm{dl}$ lower at the 36th $\mathrm{GW}(\mathrm{P}=0.036)$, among riboflavin deficient (EGRac

$\geq 1.4$ ) women compared to women with optimal riboflavin status (EGRac $\leq 1.26$ ), after controlling for known confounders. Furthermore, among women with riboflavin deficiency, compared to those sufficient in riboflavin at the 12 th GW, a significantly higher percentage went on to develop anaemia by the 36 th GW $(4.6 \%$ vs $10.6 \%, P=0.032)$.

Riboflavin deficiency is more common in pregnancy than previously recognised. Maintenance of an optimal riboflavin status in pregnancy, through improved diet or supplementation, may improve $\mathrm{Hb}$ concentrations and reduce the risk of anaemia, however randomised trials with riboflavin are required, including the ongoing OptiPREG intervention study.

\section{Acknowledgments}

The OptiPREG study is supported by the Northern Ireland Public Health Agency, HSC R\&D Division, and DSM Nutritional Products (Kaiseraugst, Switzerland) who supported a studentship for B. Duffy. Funders were not involved in the design, analysis, or interpretation of the study.

\section{References}

1. Rahman M, Abe S, Rahman M et al. (2016) Am J Clin Nutr 103,495-504.

2. Nair M, Churchill D, Robinson S et al. (2017) Br. J. Haematol 179,829-837.

3. Sirivech S, Frieden E, Osaki S (1974) Biochem J 143,311-315.

4. Powers H, Bates C, Lamb W (1985) Hum Nutr Clin Nutr 39,117-29. 